\title{
A DOUBLE COMMUTANT THEOREM FOR CONJUGATE SELFADJOINT OPERATORS
}

\author{
JAMES W. MOELLER
}

\begin{abstract}
Let $A$ be a bounded linear transformation on the complex separable Hilbert space $H$. If there is a conjugation $Q$ on $H$ such that $A=Q A^{*} Q$, we say that $A$ is conjugate selfadjoint. In this note we examine commutativity properties of conjugate selfadjoint operators which possess cyclic vectors.
\end{abstract}

1. Preliminaries. Let $H$ be a complex Hilbert space with a countably infinite basis, and let $(f, g)$ denote the inner product of two vectors in $H$. By $H \oplus H$ we mean the Hilbert space of vectors $f \oplus g$ having inner product

$$
\left(f_{1} \oplus g_{1}, f_{2} \oplus g_{2}\right)=\left(f_{1}, f_{2}\right)+\left(g_{1}, g_{2}\right) \text {. }
$$

A linear manifold is a subset which is closed under vector addition and under multiplication by complex numbers. A subspace is a linear manifold which is closed in the norm topology induced by the inner product. The smallest subspace containing the set $\cup_{n=0}^{\infty}\left\{f_{n}\right\}$ will be denoted by $\bigvee\left\{f_{n}\right\}$. If $F$ is a subset of Hilbert space, clos $F$ will denote the closure of $F$ in the norm topology and $F^{\perp}=\{g \mid(g, f)$ $=0, f \in F\}$. Whenever $A$ is a continuous linear transformation on $H$, its graph $\Gamma(A)=\{f \oplus A f \mid f \in H\}$ is a subspace of $H \oplus H$. One can easily verify that

$$
\Gamma(A)^{\perp}=\left\{A^{*}(-f) \oplus f \mid f \in H\right\},
$$

where $A^{*}$ is the adjoint of $A$. The germinal idea of representing a linear transformation through its associated graph subspace originated in the work of $\mathrm{J}$. von Neumann [1]. Hereafter we shall refer to a continuous (or bounded) linear transformation as an operator. If $A$ and $B$ are operators on $H$, we define

$$
(A \oplus B)(f \oplus g)=A f \oplus B g .
$$

The set of all operators on $H$ that commute with $A$ is called the commutant of $A$. This algebra will be denoted by $\{A\}^{\prime}$. The double commutant of $A$, designated $\{A\}^{\prime \prime}$, is the algebra of all operators on $H$ that commute with every member of $\{A\}^{\prime}$. It is self evident that $\{A\}^{\prime}$ is abelian if and only if $\{A\}^{\prime}=\{A\}^{\prime \prime}$.

A transformation $Q$ on $H$ is said to be a conjugation if $Q^{2}=I$ and $(Q f, Q g)=$ $(g, f)$ for every $f$ and $g$ in $H$. Intuitively speaking, $Q$ replaces each element of $H$ by its conjugate with respect to the "real" subspace consisting of all fixed points of $Q$ [3, p. 357]. If there is a conjugation $Q$ such that $A=Q A^{*} Q$, we shall call $A$ conjugate selfadjoint. The well-known Hankel operators [4] belong to this class.

Received by the editors July 18,1980 and, in revised form, January 12, 1981. 1980 Mathematics Subject Classification. Primary 47A05; Secondary 46J99.

Key words and phrases. Hilbert space, linear transformation, commutant, cyclic vector. 
2. Conjugate selfadjoint operators with abelian commutants. A vector $f$ in $H$ is said to be cyclic for $A$ if $\bigvee\left\{A^{n} f\right\}=H$. Our first result states that any conjugate selfadjoint operator which has a cyclic vector must also have an abelian commutant.

THEOREM 1. If $A$ is a conjugate selfadjoint operator having a cyclic vector, then $\{A\}^{\prime}=\{A\}^{\prime \prime}$.

Proof. Let $C$ be any operator in $\{A\}^{\prime}$, and let $f$ be cyclic for $A$. If $g=C f$, then $\Gamma(C)=\bigvee\left(A^{n} f \oplus A^{n} g\right\}$ because $\bigvee\left\{A^{n} f\right\}=H$. Since $A$ is conjugate selfadjoint, this implies that $\Gamma(C)=\bigvee\left(A^{n} f \oplus Q A^{* n} Q g\right\}$. But

$$
\begin{aligned}
\left(A^{k} f \oplus Q A^{* k} Q g, A^{* j} Q g \oplus Q A^{j}(-f)\right) & =\left(A^{k} f, A^{* j} Q g\right)-\left(Q A^{* k} Q g, Q A^{j} f\right) \\
& =\left(A^{k+j} f, Q g\right)-\left(A^{j+k} f, Q g\right)=0,
\end{aligned}
$$

so

$$
\bigvee\left\{A^{* n} Q g \oplus Q A^{n}(-f)\right\} \subset \Gamma(C)^{\perp}
$$

Therefore $\Gamma(Q C Q) \subset \Gamma\left(C^{*}\right)$, and it follows that $Q C Q=C^{*}$ because $-f$ is cyclic for $A$. Now let $D \in\{A\}^{\prime}$. Since $\{A\}^{\prime}$ is an algebra, $D C \in\{A\}^{\prime}$ and we have

$$
C D=Q(C D)^{*} Q=\left(Q D^{*} Q\right)\left(Q C^{*} Q\right)=D C .
$$

Thus $\{A\}^{\prime}=\{A\}^{\prime \prime}$.

COROLlaRY. If $A$ is an operator and $A=-Q A^{*} Q$ for some conjugation $Q$, then $A^{2}$ has no cyclic vectors.

Proof. Suppose that $B$ is a conjugate selfadjoint operator having a cyclic vector. The arguments used in the previous proof imply that $Q C^{*} Q=C$ whenever $C \in\{B\}^{\prime}$. The desired result follows by taking $B=A^{2}$.

3. An extension of the previous result. If $A$ and $B$ are operators on $H$, and if there is an operator $J$ such that $J A=B J$, then $J$ is said to intertwine $B$ and $A$. In the remainder of this section we shall investigate the situation which occurs when there is an intertwining operator between $Q A^{*} Q$ and $A$. To do so, we again use graph subspaces.

Following [2, p. 293], we define the strong graph limit of a sequence of operators $F_{n}$ to be the manifold

$$
\Gamma_{\infty}\left(F_{n}\right)=\left\{f \oplus g \mid f_{n} \rightarrow f \text { and } F_{n}\left(f_{n}\right) \rightarrow g\right\} .
$$

LEMMA 1. If $A$ and $B$ are operators on $H$, then $A B=B A$ if and only if $(A \oplus A) \Gamma(B) \subset \Gamma(B)$.

Proof. Obvious.

LEMMA 2. If $K$ is an operator on $H$, and if $F_{n} \in\{K\}^{\prime}$, then $\operatorname{clos} \Gamma_{\infty}\left(F_{n}\right)$ is an invariant subspace for $K \oplus K$.

Proof. Since $F_{n} \in\{K\}^{\prime}, \Gamma\left(F_{n}\right)$ is an invariant subspace for $K \oplus K$ by virtue of Lemma 1. If $f_{n} \rightarrow f$ and $F_{n}\left(f_{n}\right) \rightarrow g$, it follows that $K f_{n} \rightarrow K f$ and $F_{n} K\left(f_{n}\right)=K F_{n}\left(f_{n}\right)$ $\rightarrow K g$. Hence $(K \oplus K)\left(\Gamma_{\infty}\left(F_{n}\right)\right) \subset \Gamma_{\infty}\left(F_{n}\right)$, and the desired conclusion is obtained by taking closures. 
THEOREM 2. Let $A$ be an operator on $H$ which has a cyclic vector $f$. If there is an injective opertor $J$ whose range is dense in $H$ satisfying the property that $J A=$ $Q A^{*} Q J$ for some conjugation $Q$, then $\{A\}^{\prime}=\{A\}^{\prime \prime}$.

Proof. Let $B$ and $C$ denote operators in $\{A\}^{\prime}$. Since $J A=Q A^{*} Q J$, it follows by induction that $J p(A)=Q p(A)^{*} Q J$ whenever $p$ is a complex polynomial (see properties of conjugations in [3]). But $f$ is cyclic for $A$, so there is a sequence of polynomials $p_{n}$ such that $p_{n}(A) A^{k} f \rightarrow C A^{k} f$ for all $k=0,1, \ldots$ Therefore $A^{B} f \oplus C A^{k} f \in \Gamma_{\infty}\left(p_{n}(A)\right)$ and $p_{n}(A)^{*} A^{* k} Q J f \rightarrow Q J A^{k} C f$. Since

$$
\left(f \oplus p_{n}(A) f, p_{n}(A) *(-g) \oplus g\right)=0
$$

for all $f$ and $g$ independently of $n$, the subspace

$$
\bigvee_{j=0}^{\infty}\left\{Q J A^{j} C f \oplus A^{* j} Q J(-f)\right\}
$$

is orthogonal to $\Gamma_{\infty}\left(p_{n}(A)\right)$. Consequently

$$
\bigvee_{j=0}^{\infty}\left\{A^{* j} Q J f \oplus A^{* j} C f\right) \subset \Gamma\left(C^{*}\right)
$$

But $Q J f$ is cyclic for $A^{*}$ because $J$ is injective and has a dense range. Hence

$$
\Gamma\left(C^{*}\right)=\bigvee\left\{A^{* n} Q J f \oplus A^{* n} C f\right\}
$$

owing to the continuity of $C$, and we conclude that clos $\Gamma_{\infty}\left(p_{n}(A)\right)=\Gamma(C)$. Since $(B \oplus B) \Gamma\left(p_{n}(A)\right) \subset \Gamma\left(p_{n}(A)\right)$, it follows from Lemma 2 that $(B \oplus B) \Gamma(C) \subset \Gamma(C)$. Thus $\{A\}^{\prime}=\{A\}^{\prime \prime}$ by Lemma 1 .

Although selfadjoint linear transformations are well understood thanks to the spectral theorem, very little appears to be known about conjugate selfadjoint operators. It would be of interest to learn whether they have nontrivial invariant or hyperinvariant subspaces.

ACKNOWLeDgements. The author wishes to thank L. Gearhart, N. Rickert, W. F. Stinespring and the referee for helpful criticism.

\section{REFERENCES}

1. J. von Neumann, Über adjungierte Funktionaloperatoren, Ann. of Math. 33 (1932), pp. 294-310.

2. M. Reed and B. Simon, Methods of modern mathematical physics. Vol. I: Functional analysis, Academic Press, New York, 1972.

3. M. H. Stone, Linear transformations in Hilbert space and their applications to analysis, Amer. Math. Soc. Colloq. Publ., vol. 15, Amer. Math. Soc., Providence, R.I., 1932.

4. H. Widom, Hankel matrices, Trans. Amer. Math. Soc. 121 (1966), 1-35.

Department of Mathematics, University of Illinois at Chicago Circle, Chicago, Illinois 60680 